\title{
Correction to: Considerations in Developing Reporter Gene Bioassays for Biologics
}

\author{
Jamison Grailer, Richard A. Moravec, Zhijie Jey Cheng, \\ Manuela Grassi, Vanessa Ott, Frank Fan, and Mei Cong
}

Correction to:

Seng-Lai Tan (ed.), Immuno-Oncology: Cellular and Translational Approaches, Methods in Pharmacology and Toxicology,

https://doi.org/10.1007/978-1-0716-0171-6_9

The chapter "Considerations in Developing Reporter Gene Bioassays for Biologics" has been made open access under a CC BY 4.0 license and the Copyright Holder is now "The Author(s)". The book has also been updated to reflect this change.

The updated online version of this chapter can be found at https://doi.org/10.1007/978-1-0716-0171-6_9 